\title{
Resolution and implementation of the nonstationary vorticity velocity pressure formulation of the Navier-Stokes equations
}

\author{
Mohamed Abdelwahed ${ }^{1}$, Ebtisam Alharbi ${ }^{1}$, Nejmeddine Chorfi ${ }^{1}$ and Henda Ouertani ${ }^{2 *}$
}

\author{
"Correspondence: \\ houertani@ksu.edu.sa \\ ${ }^{2}$ Information Technology \\ Department, College of Computer \\ and Information Sciences, King \\ Saud University, Riyadh, Saudi \\ Arabia \\ Full list of author information is \\ available at the end of the article
}

\begin{abstract}
This paper deals with the iterative algorithm and the implementation of the spectral discretization of time-dependent Navier-Stokes equations in dimensions two and three. We present a variational formulation, which includes three independent unknowns: the vorticity, velocity, and pressure. In dimension two, we establish an optimal error estimate for the three unknowns. The discretization is deduced from the implicit Euler scheme in time and spectral methods in space. We present a matrix linear system and some numerical tests, which are in perfect concordance with the analysis.
\end{abstract}

Keywords: Nonstationary Navier-Stokes equations; Implicit Euler's scheme; Spectral discretization; GMRES method

\section{Introduction}

The nonlinear Navier-Stokes equations model the flow of a viscous and incompressible fluid such as water, air, and oil in stationary or nonstationary states. Those equations were and are the subject of a large number of research papers. The modification of any of the parameters associated with these equations (the domain on which the equations are posed, boundary conditions, nature of the data, variational formulation, time dependence, choice of the approximation method, etc.) leads to new research problems. In the initiating paper [1] the authors handle the Stokes and Navier-Stokes equations with nonstandard boundary conditions on the velocity and the pressure for a convex or regular domain. Our interest concerns the nonstationary Navier-Stokes equations with boundary conditions on the normal component of the velocity and the tangential components of the potential vector vorticity. Such a problem allows us to model, for instance, two fluids separated by a membrane or the flow in a network of pipes. The equivalent variational formulation of the Navier-Stokes equations provided with these boundary conditions admits three unknowns: the vorticity, velocity, and pressure [2-5]. This formulation has been studied in several works for the finite element discretization of the Stokes and Navier-Stokes problems in the stationary case (see $[3,6])$. We cite in the same context the works of Bernardi et al. $[7,8]$, which present a posteriori error analysis of time-dependent Stokes and Navier-

(c) The Author(s) 2020. This article is licensed under a Creative Commons Attribution 4.0 International License, which permits use, sharing, adaptation, distribution and reproduction in any medium or format, as long as you give appropriate credit to the original author(s) and the source, provide a link to the Creative Commons licence, and indicate if changes were made. The images or other third party material in this article are included in the article's Creative Commons licence, unless indicated otherwise in a credit line to the material. If material is not included in the article's Creative Commons licence and your intended use is not permitted by statutory regulation or exceeds the permitted use, you will need to obtain permission directly from the copyright holder. To view a copy of this licence, visit http://creativecommons.org/licenses/by/4.0/. 
Stokes problems. The extension to spectral discretization has been handled in $[9,10]$ for the stationary Stokes and Navier-Stokes problems and in [11,12] for the nonstationary case.

In this paper, we propose a discretization of such a formulation by the implicit Euler scheme in time and the spectral method in space in the square $]-1,1\left[{ }^{2}\right.$ for dimension two and in the cube $]-1,1\left[{ }^{3}\right.$ for dimension three. The spectral method can be easily extended to more complex geometries thanks to the arguments in [13, 14]. In dimension two, we prove an optimal error estimate for the vorticity and the velocity and a quasioptimal error for the pressure, using the theorem of Brezzi, Rappaz, and Raviart [15]. However, the extension to dimension three remains a difficult problem.

We describe a numerical algorithm used to solve the discrete nonlinear problem. We also present clearly the matrices and the linear system derived from the discrete problem. This linear system is solved using the GMRES method since the global matrix is not symmetric [16].

Finally, we present some numerical experiments, which confirm a good convergence of our algorithm and the benefit of this formulation. These numerical results are coherent with the theoretical ones.

This paper is organized as follows:

- In Sect. 2, we present a continuous problem and some regularity results.

- Sect. 3 is devoted to a description of time and full discrete problems.

- An error estimate is derived in Sect. 4.

- In Sect. 5, we describe an iterative algorithm used to solve a nonlinear discrete problem and a linear matrix system. We conclude by presenting some numerical experiments.

\section{A continuous problem and some regularity results}

We consider an open bounded simply connected domain $\Omega$ of $\mathbb{R}^{d}(d=2, d=3)$ with Lipschitz and connected boundary $\Gamma$. Let $\mathbf{x}=(x, y)$ for $d=2$ or $\mathbf{x}=(x, y, z)$ for $d=3$ be the Cartesian coordinates. In this paper, we mainly focus on the following nonstationary Navier-Stokes system:

$$
\begin{cases}\frac{\partial \mathbf{v}}{\partial t}(\mathbf{x}, t)-v \Delta \mathbf{v}(\mathbf{x}, t)+(\mathbf{v} \cdot \nabla \mathbf{v})(\mathbf{x}, t)+\nabla P(\mathbf{x}, t)=\mathbf{f}(\mathbf{x}, t) & \text { in } \Omega \times[0, \mathrm{~T}], \\ \operatorname{div} \mathbf{v}(\mathbf{x}, t)=0 & \text { in } \Omega \times[0, \mathrm{~T}], \\ \mathbf{v}(\mathbf{x}, t) \cdot \mathbf{n}(\mathbf{x})=0 & \text { on } \Gamma \times[0, \mathrm{~T}], \\ \varsigma(\operatorname{curl} \mathbf{v})(\mathbf{x}, t)=\mathbf{0} & \text { on } \Gamma \times[0, \mathrm{~T}], \\ \mathbf{v}(\mathbf{x}, 0)=\mathbf{v}_{0} & \text { in } \Omega,\end{cases}
$$

where $\mathbf{v}$ and $P$ are the unknowns velocity and pressure, $\mathbf{f}$ represents the density of the body forces, $v>0$ is the viscosity, and $\mathbf{n}$ is the unit outward normal vector on the boundary $\Gamma$. We define the boundary operator $\varsigma$ such that $\zeta(\operatorname{curl} \mathbf{v})$ is the boundary of curl $\mathbf{v}$ in dimension $d=2$ or the boundary of the tangential components of the curl $\mathbf{v}$ in dimension $d=3$. 
We introduce the unknown vorticity $\theta=\operatorname{curl} \mathbf{v}($ see $[2,3])$, and since $\mathbf{v} \cdot \nabla \mathbf{v}$ is equal to $\theta \times \mathbf{v}+\frac{1}{2} \operatorname{grad}|\mathbf{v}|^{2}$, system (1) is equivalent to the system

$$
\begin{cases}\frac{\partial \mathbf{v}}{\partial t}(\mathbf{x}, t)+v(\operatorname{curl} \theta)(\mathbf{x}, t)+(\theta \times \mathbf{v})(\mathbf{x}, t)+\nabla p(\mathbf{x}, t)=\mathbf{f}(\mathbf{x}, t) & \text { in } \Omega \times[0, \mathrm{~T}], \\ \operatorname{div} \mathbf{v}(\mathbf{x}, t)=0 & \text { in } \Omega \times[0, \mathrm{~T}], \\ \theta(\mathbf{x}, t)=\operatorname{curl} \mathbf{v}(\mathbf{x}, t) & \text { in } \Omega \times[0, \mathrm{~T}], \\ \mathbf{v}(\mathbf{x}, t) \cdot \mathbf{n}(\mathbf{x})=0 & \text { on } \partial \Omega \times[0, \mathrm{~T}], \\ \varsigma(\theta)(\mathbf{x}, t)=\mathbf{0} & \text { on } \partial \Omega \times[0, \mathrm{~T}], \\ \mathbf{v}(\mathbf{x}, 0)=\mathbf{v}_{0} & \text { in } \Omega .\end{cases}
$$

The dynamical pressure $p$ is defined as

$$
p=P+\frac{1}{2}|\mathbf{v}|^{2} .
$$

We assume that the following condition is satisfied by the initial velocity $\mathbf{v}_{0}$ and the initial vorticity $\theta_{0}=\theta(\mathbf{x}, 0)$ :

$$
\operatorname{div} \mathbf{v}_{0}=0 \quad \text { in } \Omega, \quad \text { and } \quad \theta_{0}=\operatorname{curl} \mathbf{v}_{0} \quad \text { in } \Omega .
$$

We define the space

$$
H(\operatorname{div}, \Omega)=\left\{\mathbf{u} \in L^{2}(\Omega)^{d} ; \operatorname{div} \mathbf{u} \in L^{2}(\Omega)\right\}
$$

equipped with the norm

$$
\|\mathbf{u}\|_{H(\operatorname{div}, \Omega)}=\left(\|\mathbf{u}\|_{L^{2}(\Omega)^{d}}^{2}+\|\operatorname{div} \mathbf{u}\|_{L^{2}(\Omega)}^{2}\right)^{\frac{1}{2}}
$$

and its subspace

$$
H_{0}(\operatorname{div}, \Omega)=\{\mathbf{u} \in H(\operatorname{div}, \Omega) ; \mathbf{u} . \mathbf{n}=0 \text { on } \Gamma\} .
$$

We also introduce the space

$$
H(\operatorname{curl}, \Omega)=\left\{\varphi \in L^{2}(\Omega)^{d} ; \operatorname{curl} \varphi \in L^{2}(\Omega)^{\frac{d(d-1)}{2}}\right\}
$$

provided with the norm

$$
\|\mathbf{u}\|_{H(\mathbf{c u r l}, \Omega)}=\left(\|\mathbf{u}\|_{L^{2}(\Omega)^{d}}^{2}+\|\operatorname{curl} \mathbf{u}\|_{L^{2}(\Omega)}^{2} \frac{d(d-1)}{2}\right)^{\frac{1}{2}}
$$

and its subspace

$$
H_{0}(\operatorname{curl}, \Omega)=\{\mathbf{u} \in H(\operatorname{curl}, \Omega) ; \varsigma(\mathbf{u})=0 \text { on } \Gamma\} .
$$

We recall that

$$
L_{0}^{2}(\Omega)=\left\{u \in L^{2}(\Omega) ; \int_{\Omega} u d x=0\right\}
$$

and $(\cdot, \cdot)$ is the $L^{2}(\Omega)$ scalar product. 
Let $B$ a separable Banach space. We need to define the following space to handle the nonstationary Navier-Stokes system:

$$
L^{p}(0, T ; B)=\{\mathbf{u} \text { measurable on }] 0, T\left[\text { such that } \int_{0}^{T}\|\mathbf{u}(t, \cdot)\|_{B}^{p} d t<\infty\right\},
$$

which is a Banach space with the norm

$$
\|\mathbf{u}\|_{L^{p}(0, T ; B)}= \begin{cases}\left(\int_{0}^{T}\|\mathbf{u}(t, \cdot)\|_{B}^{p} d t\right)^{\frac{1}{p}}, & \text { for } 1 \leq p<+\infty \\ \sup _{0 \leq t \leq T}\|\mathbf{u}(t, \cdot)\|_{B}, & \text { for } p=+\infty\end{cases}
$$

We also introduce the Banach space $\mathcal{L}(B)$ of the continuous linear functions from $B$ to $\mathbb{R}$ with the norm

$$
\forall L \in \mathcal{L}(B), \quad\|L\|_{\mathcal{L}(B)}=\sup _{x \in B /\{0\}} \frac{|L(x)|}{\|x\|_{B}}
$$

If the data $\mathbf{f}$ belongs to the space $L^{2}\left(0, T ; H_{0}(\operatorname{div}, \Omega)^{\prime}\right)$, where $\left(H_{0}(\operatorname{div}, \Omega)^{\prime}\right.$ is the dual space of $H_{0}(\operatorname{div}, \Omega)$ (see [17] for more detail ), then problem (2) is equivalent to the following variational formulation:

Find $(\theta, \mathbf{v}, p) \in L^{2}\left(0, T ; H_{0}(\operatorname{curl}, \Omega)\right) \times L^{2}\left(0, T ; H_{0}(\operatorname{div}, \Omega)\right) \times L^{2}\left(0, T ; L_{0}^{2}(\Omega)\right)$ such that

$$
\begin{cases}\forall \mathbf{w} \in H_{0}(\operatorname{div}, \Omega), & \left(\frac{\partial \mathbf{v}}{\partial t}, \mathbf{w}\right)+l(\theta, \mathbf{v} ; \mathbf{w})+Z(\theta, \mathbf{v} ; \mathbf{w})+b(\mathbf{w}, p)=\prec \mathbf{f}, \mathbf{w} \succ \\ \forall q \in L_{0}^{2}(\Omega), & b(\mathbf{v}, q)=0 \\ \forall \boldsymbol{\vartheta} \in H_{0}(\operatorname{curl}, \Omega), & t(\theta, \mathbf{v} ; \boldsymbol{\vartheta})=0\end{cases}
$$

where $\prec \cdot, \cdot \succ$ is the duality product between $H_{0}(\operatorname{div}, \Omega)^{\prime}$ and $H_{0}(\operatorname{div}, \Omega)$. The bilinear forms $l(\cdot, \cdot ; \cdot), b(\cdot, \cdot)$ and $t(\cdot, \cdot ; \cdot)$ are defined as follows:

$$
\begin{aligned}
& l(\theta, \mathbf{v} ; \mathbf{w})=v \int_{\Omega}(\operatorname{curl} \theta)(\mathbf{x}, t) \cdot \mathbf{w}(\mathbf{x}) d \mathbf{x}, \quad b(\mathbf{v}, q)=-\int_{\Omega} \operatorname{div} \mathbf{v}(\mathbf{x}, t) q(\mathbf{x}) d \mathbf{x}, \\
& t(\theta, \mathbf{v} ; \boldsymbol{\vartheta})=\int_{\Omega} \theta(\mathbf{x}, t) . \vartheta(\mathbf{x}) d \mathbf{x}-\int_{\Omega} \mathbf{v}(\mathbf{x}, t) \cdot \operatorname{curl} \boldsymbol{\vartheta}(\mathbf{x}) d \mathbf{x} .
\end{aligned}
$$

In another way, we define the nonlinear term $Z(\cdot, \cdot ; \cdot)$ by

$$
Z(\theta, \mathbf{v} ; \mathbf{w})=\int_{\Omega}(\theta \times \mathbf{v})(\mathbf{x}, t) \cdot \mathbf{w}(\mathbf{x}) d \mathbf{x} .
$$

For proving the existence of solution for problem (2), we need to define the following two kernels:

$$
\mathrm{K}=\left\{\boldsymbol{\varphi} \in H_{0}(\operatorname{div}, \Omega) ; \forall q \in L_{0}^{2}(\Omega), b(\varphi, q)=0\right\},
$$

the kernel of the bilinear form $b(\cdot, \cdot)$, which coincides with the space of divergence-free functions in $H_{0}(\operatorname{div}, \Omega)$, and

$$
\begin{aligned}
\mathrm{W} & =\left\{(\boldsymbol{\vartheta}, \boldsymbol{\varphi}) \in H_{0}(\operatorname{curl}, \Omega) \times \mathrm{K} ; \forall \boldsymbol{\psi} \in H_{0}(\operatorname{curl}, \Omega), t(\boldsymbol{\vartheta}, \boldsymbol{\varphi} ; \boldsymbol{\psi})=0\right\} \\
& =\left\{(\boldsymbol{\vartheta}, \boldsymbol{\varphi}) \in H_{0}(\operatorname{curl}, \Omega) \times \mathrm{K} ; \boldsymbol{\varphi}=\operatorname{curl} \boldsymbol{\vartheta}\right\}
\end{aligned}
$$


the kernel of the bilinear form $t(\cdot, \cdot ; \cdot)$. From the continuity of the bilinear forms $b(\cdot, \cdot)$ and $t(\cdot, \cdot ; \cdot)$ we deduce that $\mathrm{K}$ and $\mathrm{W}$ are Hilbert spaces.

If $(\theta, \mathbf{v}, p)$ is a solution of problem (4), then $(\theta, \mathbf{v})$ is solution of the following reduced problem:

Find $(\theta, \mathbf{v}) \in L^{2}(0, T ; \mathrm{W})$ such that

$$
\forall \mathbf{w} \in \mathrm{K}, \quad\left(\frac{\partial \mathbf{v}}{\partial t}, \mathbf{w}\right)+l(\theta, \mathbf{v} ; \mathbf{w})+Z(\theta, \mathbf{v} ; \mathbf{w})=\prec \mathbf{f}, \mathbf{w} \succ .
$$

In dimension two, it is simple to show that problem (5) has a solution. However, in dimension three, giving a sense to the nonlinear term $Z(\cdot, \cdot ; \cdot)$ is related to the following Assumption 1 . In that case, the spaces $H_{0}(\operatorname{div}, \Omega) \cap H(\operatorname{curl}, \Omega)$ and $H(\operatorname{div}, \Omega) \cap H_{0}(\operatorname{curl}, \Omega)$ are compactly embedded in $H^{1}(\Omega)$ see ([18], Thm 2.17).

Assumption 1 In dimension three, we suppose that the boundary $\Gamma$ is $\mathcal{C}^{1,1}$ or convex.

We recall the uniform inf-sup condition on bilinear form $b(\cdot, \cdot)$ :

There exists a constant $\gamma>0$ such that

$$
\forall q \in L_{0}^{2}(\Omega), \quad \sup _{\mathbf{u} \in H_{0}(\operatorname{div}, \Omega)} \frac{b(\mathbf{u}, q)}{\|\mathbf{u}\|_{H(\operatorname{div}, \Omega)}} \geq \gamma\|q\|_{L^{2}(\Omega)} l ;
$$

see [19] or [20, Chap. I, Cor. 2.4] for its proof.

When Assumption 1 and the inf-sup condition (6) are satisfied, then problems (5) and (4) have a solution; see [21, Chap. III, Thm. 1.1], [22, Chap. V], and [9] for the proof.

Finally, we establish some regularity properties of the solution of problem (4). These regularity results can be easily derived from [18, Chap. 2], [23], and [24] by using a bootstrap argument.

When $\Omega$ is convex and $\mathbf{f}$ belongs to $L^{2}\left(0, T ; H^{\max (0, s-1)}(\Omega)^{d}\right)$, the solution $(\theta, \mathbf{v}, p)$ belongs to the space $L^{2}\left(0, T ; H^{s}(\Omega)^{\frac{d(d-1)}{2}}\right) \times L^{2}\left(0, T ; H^{s}(\Omega)^{d}\right) \times L^{2}\left(0, T ; H^{s}(\Omega)\right)$ for all $s \leq 1$, whereas a greater regularity of the solution holds in dimension two [25, 26]:

If $\mathbf{f}$ belongs to $L^{2}\left(0, T ; H^{\max (0, s)}(\Omega)^{2}\right)$, then the solution $(\theta, \mathbf{v}, p)$ belongs to the space

$L^{2}\left(0, T ; H^{s+1}(\Omega)\right) \times L^{2}\left(0, T ; H^{s}(\Omega)^{2}\right) \times L^{2}\left(0, T ; H^{s+1}(\Omega)\right)$ for all

i) $s \leq \frac{1}{2}$ in the general case,

ii) $s \leq 1$ when $\Omega$ is convex, and

iii) $s \leq \frac{\pi}{\omega}$ when $\Omega$ is a polygon with the largest angle equal to $\omega$.

\section{The time and full discrete problems}

For the time discretization of the nonstationnary Navier-Stokes problem, we use the backward Euler method. We start by doing a partition of the interval $[0, T]$ in subintervals $\left[t_{i-1}, t_{i}\right]$ for $1 \leq i \leq I$, where $I$ is a positive integer such that $0=t_{0}<t_{1}<\cdots<t_{I}=T$. Let $h_{i}=t_{i}-t_{i-1}, h=\left(h_{1}, h_{2}, \ldots, h_{I}\right)$ and $|h|=\max _{1 \leq i \leq I} h_{i}$.

If the data $\mathbf{f}$ belongs to the space $L^{2}\left(0, T ;\left(H_{0}(\operatorname{div}, \Omega)\right)^{\prime}\right)$ and $\left(\theta_{0}, \mathbf{v}_{0}\right) \in H_{0}(\operatorname{curl}, \Omega) \times$ $H_{0}(\operatorname{div}, \Omega)$ satisfy condition (3), then the time semidiscrete problem is:

Find $\left(\theta^{i}\right)_{0 \leq i \leq I} \in\left(H_{0}(\operatorname{curl}, \Omega)\right)^{I+1},\left(\mathbf{v}^{i}\right)_{0 \leq i \leq I} \in\left(H_{0}(\operatorname{div}, \Omega)\right)^{I+1}$, and $\left(p^{i}\right)_{1 \leq i \leq I} \in\left(L_{0}^{2}(\Omega)\right)^{I}$ such that

$$
\theta^{0}=\theta_{0} \quad \text { and } \quad \mathbf{v}^{0}=\mathbf{v}_{0} \quad \text { in } \Omega
$$


and for all $1 \leq i \leq I$,

$$
\begin{aligned}
& \forall \mathbf{w} \in H_{0}(\operatorname{div}, \Omega), \hat{l}\left(\theta^{i}, \mathbf{v}^{i} ; \mathbf{w}\right)+h_{i} Z\left(\theta^{i}, \mathbf{v}^{i} ; \mathbf{w}\right)+h_{i} b\left(\mathbf{w}, p^{i}\right)=L(\mathbf{w}), \\
& \forall q \in L_{0}^{2}(\Omega), \quad b\left(\mathbf{v}^{i}, q\right)=0, \\
& \forall \vartheta \in H_{0}(\operatorname{curl}, \Omega), \quad t\left(\theta^{i}, \mathbf{v}^{i} ; \boldsymbol{\vartheta}\right)=0, \\
& \operatorname{re}^{i}=\mathbf{f}\left(\cdot, t_{i}\right), \\
& \hat{l}\left(\theta^{i}, \mathbf{v}^{i} ; \mathbf{w}\right)=\left(\mathbf{v}^{i}, \mathbf{w}\right)+h_{i} l\left(\theta^{i}, \mathbf{v}^{i} ; \mathbf{w}\right),
\end{aligned}
$$

where $\mathbf{f}^{i}=\mathbf{f}\left(\cdot, t_{i}\right)$,

and

$$
L(\mathbf{w})=\left(\mathbf{v}^{i-1}, \mathbf{w}\right)+h_{i} \prec \mathbf{f}^{i}, \mathbf{w} \succ .
$$

So when $\left(\theta^{i}, \mathbf{v}^{i}, p^{i}\right)$ is a solution of problem (7)-(8), the couple $\left(\theta^{i}, \mathbf{v}^{i}\right) \in \mathrm{W}$ is a solution of the problem

$$
\forall \mathbf{w} \in \mathrm{K}, \quad \hat{l}\left(\theta^{i}, \mathbf{v}^{i} ; \mathbf{w}\right)+h_{i} Z\left(\theta^{i}, \mathbf{v}^{i} ; \mathbf{w}\right)=L(\mathbf{w}) .
$$

The existence of a solution for problems (9) and (8) is deduced from the properties (positivity and inf-sup conditions) of the bilinear form $\hat{l}(\cdot, \cdot ; \cdot)$ proved in [11, Lemma 1], the properties of the trilinear form $Z(\cdot, \cdot ; \cdot)$ (continuity and antisymmetry) proved in [12, Lemma 1], and the inf-sup condition (6). We summarize this result of the existence in dimensions two and three in the following theorem; see [12, Sect. 3], for its proof.

Theorem 1 Suppose the data $\mathbf{f} \in L^{2}\left(0, t ;\left(H_{0}(\operatorname{div}, \Omega)\right)^{\prime}\right)$ and the initial vorticity-velocity $\left(\theta_{0}, \mathbf{v}_{0}\right)$ both belong to $H_{0}(\mathrm{curl}, \Omega) \times H_{0}(\mathrm{div}, \Omega)$ and satisfy condition (3). In dimensiond $=2$, for any $i, 1 \leq i \leq I$, problem (7)-(8) has a solution $\left(\theta^{i}, \mathbf{v}^{i}, p^{i}\right)$ in $H_{0}(\operatorname{curl}, \Omega) \times H_{0}(\operatorname{div}, \Omega) \times$ $L^{2}(\Omega)$. In dimension $d=3$, if Assumption 1 holds and there exists a constant $\varrho$ such that

$$
\varrho v^{-2}\|L\|_{\mathcal{L}\left(H_{0}(\mathrm{div}, \Omega)\right)}<1
$$

then problem (7)-(8) has a solution $\left(\theta^{i}, \mathbf{v}^{i}, p^{i}\right)$ in $H_{0}(\operatorname{curl}, \Omega) \times H_{0}(\operatorname{div}, \Omega) \times L^{2}(\Omega)$. The pair $\left(\theta^{i}, \mathbf{v}^{i}\right)$ satisfies the following stability condition:

$$
\sum_{j=1}^{i}\left\|\theta^{j}\right\|_{H(\mathbf{c u r l}, \Omega)}^{2}+\left\|\mathbf{v}^{i}\right\|_{L^{2}(\Omega)^{d}}^{2} \leq \frac{c}{v}\left(\left\|\mathbf{v}_{0}\right\|_{L^{2}(\Omega)^{d}}^{2}+\sum_{j=1}^{i} h_{j}\left\|\mathbf{f}^{j}\right\|_{H_{0}(\mathrm{div}, \Omega)^{\prime}}^{2}\right),
$$

where $c$ is a positive constant independent of $i$.

Hereinafter, for the spectral discretization, we assume that $\Omega$ is a square or cube. Using the same idea of Nédélec's finite elements (see [27, Sect. 2]), we introduce our discrete spaces.

Let $N \geq 2$ be an integer. The velocity discrete space $\mathbb{V}_{N}$ is defined as

$$
\mathbb{V}_{N}=H_{0}(\operatorname{div}, \Omega) \cap \begin{cases}\mathbb{P}_{N, N-1}(\Omega) \times \mathbb{P}_{N-1, N}(\Omega) & \text { if } d=2, \\ \mathbb{P}_{N, N-1, N-1}(\Omega) \times \mathbb{P}_{N-1, N, N-1}(\Omega) \times \mathbb{P}_{N-1, N-1, N}(\Omega) & \text { if } d=3\end{cases}
$$


The vorticity discrete space $\mathbb{T}_{N}$ is defined as

$$
\mathbb{T}_{N}= \begin{cases}H_{0}^{1}(\Omega) \cap \mathbb{P}_{N}(\Omega) & \text { if } d=2, \\ H_{0}(\mathbf{c u r l}, \Omega) \cap\left(\mathbb{P}_{N-1, N, N}(\Omega) \times \mathbb{P}_{N, N-1, N}(\Omega) \times \mathbb{P}_{N, N, N-1}(\Omega)\right) & \text { if } d=3\end{cases}
$$

Finally, the pressure discrete spaces $\mathbb{M}_{N}$ are defined as

$$
\mathbb{M}_{N}=L_{0}^{2}(\Omega) \cap \mathbb{P}_{N-1}(\Omega)
$$

Let the nodes $\epsilon_{i}, 0 \leq i \leq N$, be the zeros of the polynomial $\left(1-x^{2}\right) L_{N}^{\prime}$, where $L_{N}$ is the Legendre polynomial of degree $N$ on the interval $[-1,1]$, and let $\rho_{i}, 0 \leq i \leq N$, be the set of weights for the Gauss-Lobatto quadrature formula. Then

$$
\forall \varphi \in \mathbb{P}_{2 N-1}(-1,1), \quad \int_{-1}^{1} \varphi(\zeta) d \zeta=\sum_{j=0}^{N} \varphi\left(\epsilon_{j}\right) \rho_{j}
$$

We have the following inequality [28]:

$$
\forall u_{N} \in \mathbb{P}_{N}(-1,1), \quad\left\|u_{N}\right\|_{L^{2}(-1,1)}^{2} \leq \sum_{k=0}^{N} u_{N}^{2}\left(\epsilon_{k}\right) \rho_{k} \leq 3\left\|u_{N}\right\|_{L^{2}(-1,1)}^{2} .
$$

For continuous functions $\varphi$ and $\psi$ on $\bar{\Omega}$, we define the discrete scalar product

$$
(\varphi, \psi)_{N}= \begin{cases}\sum_{k=0}^{N} \sum_{l=0}^{N} \varphi\left(\epsilon_{k}, \epsilon_{l}\right) \psi\left(\epsilon_{k}, \epsilon_{l}\right) \rho_{k} \rho_{l} & \text { if } d=2, \\ \sum_{k=0}^{N} \sum_{l=0}^{N} \sum_{r=0}^{N} \varphi\left(\epsilon_{k}, \epsilon_{l}, \epsilon_{r}\right) \psi\left(\epsilon_{k}, \epsilon_{l}, \epsilon_{r}\right) \rho_{k} \rho_{l} \rho_{r} & \text { if } d=3\end{cases}
$$

Hereinafter, we suppose that $\mathbf{f}$ is continuous on $\bar{\Omega} \times[0, T]$. The full discrete problem is constructed from problem (7)-(8) by using the Galerkin method combined with numerical integration.

If $\mathbf{v}_{N}^{0}=\mathrm{I}_{N}\left(\mathbf{v}_{0}\right)$, then knowing $\mathbf{v}_{N}^{i-1}$, we find $\left(\boldsymbol{\tau}_{N}^{i}, \mathbf{v}_{N}^{i}, p_{N}^{i}\right)$ in $\mathbb{T}_{N} \times \mathbb{V}_{N} \times \mathbb{M}_{N}$ such that for $1 \leq i \leq I$,

$$
\begin{aligned}
& \forall \mathbf{w}_{N} \in \mathbb{V}_{N}, \quad \hat{l}_{N}\left(\theta_{N}^{i}, \mathbf{v}_{N}^{i} ; \mathbf{w}_{N}\right)+h_{i} Z_{N}\left(\theta_{N}^{i}, \mathbf{v}_{N}^{i} ; \mathbf{w}_{N}\right)+h_{i} b_{N}\left(\mathbf{w}_{N}, p_{N}^{i}\right)=L_{N}\left(\mathbf{w}_{N}\right), \\
& \forall q_{N} \in \mathbb{M}_{N}, \quad b_{N}\left(\mathbf{v}_{N}^{i}, q_{N}\right)=0, \\
& \forall \boldsymbol{\vartheta}_{N} \in \mathbb{T}_{N}, \quad t_{N}\left(\theta_{N}^{i}, \mathbf{v}_{N}^{i} ; \boldsymbol{\vartheta}_{N}\right)=0 .
\end{aligned}
$$

The bilinear forms $\hat{l}_{N}(\cdot, \cdot ; \cdot), b_{N}(\cdot, \cdot)$, and $t_{N}(\cdot, \cdot ; \cdot)$ are defined as follows:

$$
\begin{aligned}
& \hat{l}_{N}\left(\theta_{N}^{i}, \mathbf{v}_{N}^{i} ; \mathbf{w}_{N}\right)=\left(\mathbf{v}_{N}^{i}, \mathbf{w}_{N}\right)_{N}+h_{i} v\left(\operatorname{curl} \theta_{N}^{i}, \mathbf{w}_{N}\right)_{N}, \quad b_{N}\left(\mathbf{w}_{N}, q_{N}\right)=-\left(\operatorname{div} \mathbf{w}_{N}, q_{N}\right)_{N}, \\
& \quad \text { and } t_{N}\left(\theta_{N}^{i}, \mathbf{v}_{N}^{i} ; \boldsymbol{\varphi}_{N}\right)=\left(\theta_{N}^{i}, \boldsymbol{\varphi}_{N}\right)_{N}-\left(\mathbf{v}_{N}^{i}, \operatorname{curl} \boldsymbol{\varphi}_{N}\right)_{N} .
\end{aligned}
$$

From (11) combined with the Cauchy-Schwarz inequality it follows that the bilinear forms $\hat{l}_{N}(\cdot, \cdot ; \cdot), b_{N}(\cdot, \cdot)$. and $t_{N}(\cdot, \cdot ; \cdot)$ are continuous respectively on $\left(\mathbb{T}_{N} \times \mathbb{V}_{N}\right) \times \mathbb{V}_{N}$, $\mathbb{V}_{N} \times \mathbb{M}_{N}$, and $\left(\mathbb{T}_{N} \times \mathbb{V}_{N}\right) \times \mathbb{T}_{N}$ with norms bounded independently of $N$. The functional 
$L_{N}\left(\mathbf{w}_{N}\right)=\left(\mathbf{v}_{N}^{i-1}, \mathbf{w}_{N}\right)_{N}+h_{i}\left(\mathrm{I}_{N}\left(\mathbf{f}^{i}\right), \mathbf{w}_{N}\right)_{N}$ is linear and continuous on $\mathbb{V}_{N}$. As a consequence of the exactness property (10), the bilinear forms $b(\cdot, \cdot)$ and $b_{N}(\cdot, \cdot)$ coincide on $\mathbb{V}_{N} \times \mathbb{M}_{N}$. The discrete nonlinear term $Z_{N}(\cdot, \cdot ; \cdot)$ is defined as

$$
Z_{N}\left(\theta_{N}^{i}, \mathbf{v}_{N}^{i} ; \mathbf{w}_{N}\right)=\left(\theta_{N}^{i} \times \mathbf{v}_{N}^{i}, \mathbf{w}_{N}\right)_{N}
$$

We introduce the kernel of the discrete bilinear form $b_{N}(\cdot, \cdot)$

$$
\mathrm{K}_{N}=\left\{\mathbf{w}_{N} \in \mathbb{V}_{N} ; \forall q_{N} \in \mathbb{M}_{N}, b_{N}\left(\mathbf{w}_{N}, q_{N}\right)=0\right\},
$$

which is equal to the space of divergence-free polynomials in $\mathbb{D}_{N}$.

We also define the discrete kernel of the bilinear form $t_{N}(\cdot, \cdot ; \cdot)$

$$
\mathrm{W}_{N}=\left\{\left(\boldsymbol{\vartheta}_{N}, \mathbf{v}_{N}\right) \in \mathbb{T}_{N} \times \mathrm{K}_{N} ; \forall \varphi_{N} \in \mathbb{T}_{N}, t_{N}\left(\vartheta_{N}, \mathbf{v}_{N} ; \varphi_{N}\right)=0\right\}
$$

We remark that the discrete kernel $\mathrm{W}_{N}$ is not included in the continuous kernel W; see [10, Cor 3.2],. So the full discrete problem (12) is reduced as follows:

If $\mathbf{v}_{N}^{0}=\mathrm{I}_{N}\left(\mathbf{v}_{0}\right)$, then knowing $\mathbf{v}_{N}^{i-1}$, find $\left(\theta_{N}^{i}, \mathbf{v}_{N}^{i}\right) \in \mathrm{W}_{N}$ such that for $1 \leq i \leq I$,

$$
\forall \mathbf{w}_{N} \in \mathrm{K}_{N}, \hat{l}_{N}\left(\theta_{N}^{i}, \mathbf{v}_{N}^{i} ; \mathbf{w}_{N}\right)+h_{i} Z_{N}\left(\theta_{N}^{i}, \mathbf{v}_{N}^{i} ; \mathbf{w}_{N}\right)=L_{N}\left(\mathbf{w}_{N}\right)
$$

We consider the inf-sup condition proved in [10, Lemma 3.9]. There exists a positive constant $\beta$ independent of $N$ such that the discrete bilinear form $b_{N}(\cdot, \cdot)$ satisfies

$$
\forall q_{N} \in \mathbb{M}_{N}, \quad \sup _{\mathbf{v}_{N} \in \mathbb{V}_{N}} \frac{b_{N}\left(\mathbf{v}_{N}, q_{N}\right)}{\left\|\mathbf{v}_{N}\right\|_{H(\operatorname{div}, \Omega)}} \geq \beta\left\|q_{N}\right\|_{L^{2}(\Omega)} .
$$

The arguments used to prove the existence of a solution of problems (13) and (12) are exactly the same as those for the continuous problems (9) and (8). These arguments are based on Brouwer's fixed point theorem [20, Chap. IV, Cor. 1.1] and the inf-sup condition (14). We summarize this result on the existence in the following theorem proved in [12, Sect. 4]

Theorem 3.1 Assume that the data $\mathbf{f}$ is continuous on $\bar{\Omega} \times[0, T]$. Then, knowing $\mathbf{v}_{N}^{i-1}$ at each time step i, problem (12) has a solution $\left(\theta_{N}^{i}, \mathbf{v}_{N}^{i}, p_{N}^{i}\right)$ in $\mathbb{T}_{N} \times \mathbb{V}_{N} \times \mathbb{M}_{N}$. Moreover, the pair $\left(\theta_{N}^{i}, \mathbf{v}_{N}^{i}\right)$ of this solution satisfies

$$
\sum_{j=1}^{i}\left\|\theta_{N}^{j}\right\|_{H(\mathbf{c u r l}, \Omega)}^{2}+\left\|\mathbf{v}_{N}^{i}\right\|_{L^{2}(\Omega)^{d}}^{2} \leq \frac{3^{d} c}{2 v}\left(\left\|\mathbf{v}_{N}^{0}\right\|_{L^{2}(\Omega)^{d}}^{2}+\sum_{j=1}^{i} h_{j}\left\|\mathrm{I}_{N}\left(\mathbf{f}^{j}\right)\right\|_{L^{2}(\Omega)^{d}}^{2}\right),
$$

where $c$ is a positive constant independent of $N$ and $i$.

Remark 1 Note that the previous existence result still holds when $Z_{N}(\cdot, \cdot ; \cdot)$ is replaced by $Z(\cdot, \cdot ; \cdot)$ in problem (12). In practice, this means that a more precise quadrature formula, exact on $\mathbb{P}_{3 N-1}(\Omega)$, is used to evaluate the integrals that appear in the treatment of the nonlinear term. The corresponding discrete problem reads: 
If $\mathbf{v}_{N}^{0}=\mathrm{I}_{N}\left(\mathbf{v}_{0}\right)$, then knowing $\mathbf{v}_{N}^{i-1}$, find $\left(\theta_{N}^{i}, \mathbf{v}_{N}^{i}, p_{N}^{i}\right)$ in $\mathbb{T}_{N} \times \mathbb{V}_{N} \times \mathbb{M}_{N}$ such that for $1 \leq$ $i \leq I$,

$$
\begin{array}{ll}
\forall \mathbf{w}_{N} \in \mathbb{V}_{N}, \quad \hat{l}_{N}\left(\theta_{N}^{i}, \mathbf{v}_{N}^{i} ; \mathbf{w}_{N}\right)+h_{i} Z\left(\theta_{N}^{i}, \mathbf{v}_{N}^{i} ; \mathbf{w}_{N}\right)+h_{i} b_{N}\left(\mathbf{w}_{N}, p_{N}^{i}\right)=L_{N}\left(\mathbf{w}_{N}\right), \\
\forall q_{N} \in \mathbb{M}_{N}, \quad b_{N}\left(\mathbf{v}_{N}^{i}, q_{N}\right)=0, \\
\forall \boldsymbol{\vartheta}_{N} \in \mathbb{T}_{N}, \quad t_{N}\left(\theta_{N}^{i}, \mathbf{v}_{N}^{i} ; \boldsymbol{\vartheta}_{N}\right)=0 .
\end{array}
$$

In the same way the discrete reduced problem (13) is written as:

If $\mathbf{v}_{N}^{0}=\mathrm{I}_{N}\left(\mathbf{v}_{0}\right)$, then knowing $\mathbf{v}_{N}^{i-1}$, find $\left(\theta_{N}^{i}, \mathbf{v}_{N}^{i}\right) \in \mathrm{W}_{N}$, such that for $1 \leq i \leq I$,

$$
\forall \mathbf{w}_{N} \in \mathrm{K}_{N}, \hat{l}_{N}\left(\theta_{N}^{i}, \mathbf{v}_{N}^{i} ; \mathbf{w}_{N}\right)+h_{i} Z\left(\theta_{N}^{i}, \mathbf{v}_{N}^{i} ; \mathbf{w}_{N}\right)=L_{N}\left(\mathbf{w}_{N}\right) .
$$

\section{Error estimates}

This section is devoted to the proof of the error estimate between the solution of problems (7)-(8) and (15) in dimension two since the proof is difficult in dimension three. This proof is based on the Brezzi-Rappaz-Raviart theorem [15].

For any data $\mathbf{f} \in L^{2}\left(0, t ; H_{0}(\operatorname{div}, \Omega)^{\prime}\right)$, we define the linear Stokes operator $\mathbb{S}$ (see [10, Cor. 2.4]), where $\mathbb{S} L$ is the solution $\left(\theta^{i}, \mathbf{v}^{i}\right)$ of the following reduced problem: Let

$$
\theta^{0}=\theta_{0} \quad \text { and } \quad \mathbf{v}^{0}=\mathbf{v}_{0} \quad \text { in } \Omega \text {. }
$$

For all $1 \leq i \leq I$, knowing $\mathbf{v}^{i-1}$, find $\left(\theta^{i}, \mathbf{v}^{i}\right) \in H_{0}(\operatorname{curl}, \Omega) \times H_{0}(\operatorname{div}, \Omega)$ such that

$$
\forall \mathbf{w} \in H_{0}(\operatorname{div}, \Omega), \hat{l}\left(\theta^{i}, \mathbf{v}^{i} ; \mathbf{w}\right)=L(\mathbf{w})
$$

We also define the mapping $\mathbb{G}$ from the space $\mathbb{X}=H_{0}(\operatorname{curl}, \Omega) \times \mathrm{K}$ into the dual space of $H_{0}(\operatorname{div}, \Omega)$ by

$$
\begin{aligned}
& \forall\left(\theta^{i}, \mathbf{v}^{i}\right) \in \mathbb{X}, 1 \leq i \leq I, \forall \mathbf{w} \in H_{0}(\operatorname{div}, \Omega) \\
& \prec \mathbb{G}\left(\theta^{i}, \mathbf{v}^{i}\right), \mathbf{w} \succ=h_{i} Z\left(\theta^{i}, \mathbf{v}^{i} ; \mathbf{w}\right)-L(\mathbf{w}) .
\end{aligned}
$$

So we conclude that problem (9) is equivalent to the problem

$$
\left(\theta^{i}, \mathbf{v}^{i}\right)+\mathbb{S} \mathbb{G}\left(\theta^{i}, \mathbf{v}^{i}\right)=0 .
$$

We proceed in the same way for the discrete case. Let $\mathbb{X}_{N}=\mathrm{W}_{N} \times \mathrm{K}_{N}$. Consider the discrete Stokes operator $\mathbb{S}_{N}$ such that $\mathbb{S}_{N} L$ is the solution $\left(\theta_{N}^{i}, \mathbf{v}_{N}^{i}\right)$ of the following problem: If $\mathbf{v}_{N}^{0}=\mathrm{I}_{N}\left(\mathbf{v}_{0}\right)$, then knowing $\mathbf{v}_{N}^{i-1}$, find $\left(\theta_{N}^{i}, \mathbf{v}_{N}^{i}\right) \in \mathrm{W}_{N}$ such that for $1 \leq i \leq I$,

$$
\forall \mathbf{w}_{N} \in \mathrm{K}_{N}, \hat{l}_{N}\left(\theta_{N}^{i}, \mathbf{v}_{N}^{i} ; \mathbf{w}_{N}\right)=L_{N}\left(\mathbf{w}_{N}\right)
$$

We also remind from [10] the following properties of the discrete operator $\mathbb{S}_{N}$ :

- The stability property

$$
\left\|\mathbb{S}_{N} L\right\|_{\mathbb{X}} \leq c \sup _{\mathbf{w}_{N} \in \mathrm{K}_{N}} \frac{\left|L\left(\mathbf{w}_{N}\right)\right|}{\left\|\mathbf{w}_{N}\right\|_{L^{2}(\Omega)^{d}}} .
$$


- If $\left(\theta^{i}, \mathbf{v}^{i}\right)$ belongs to $H^{s+1}(\Omega) \times H^{s}(\Omega)^{2}$ for any $1 \leq i \leq I$ and $s \geq 1$, then we have the following error:

$$
\left\|\left(\mathbb{S}-\mathbb{S}_{N}\right) L\right\| \leq c N^{-s}\left\|\left(\theta^{i}, \mathbf{v}^{i}\right)\right\|_{H^{s+1}(\Omega) \times H^{s}(\Omega)^{2}} .
$$

We also define the discrete mapping $\mathbb{G}_{N}$ from $\mathbb{X}_{N}$ into the dual space of $\mathbb{V}_{N}$ by

$$
\begin{aligned}
& \forall\left(\theta_{N}^{i}, \mathbf{v}_{N}^{i}\right) \in \mathbb{X}_{N}, 1 \leq i \leq I, \forall \mathbf{w}_{N} \in \mathbb{V}_{N}, \\
& \prec \mathbb{G}_{N}\left(\theta_{N}^{i}, \mathbf{v}_{N}^{i}\right), \mathbf{w}_{N} \succ=h_{i} Z\left(\theta_{N}^{i}, \mathbf{v}_{N}^{i} ; \mathbf{w}_{N}\right)-L_{N}\left(\mathbf{w}_{N}\right) .
\end{aligned}
$$

Then we conclude that the problem (13) is equivalent to the problem

$$
\left(\theta_{N}^{i}, \mathbf{v}_{N}^{i}\right)+\mathbb{S}_{N} \mathbb{G}_{N}\left(\theta_{N}^{i}, \mathbf{v}_{N}^{i}\right)=0
$$

Let $D$ be a differential operator. We make the following assumption.

Assumption 2 Knowing $\mathbf{v}^{i-1},\left(\theta^{i}, \mathbf{v}^{i}\right)$ is a solution of problem (9) such that the operator $\mathrm{Id}+\mathbb{S} D \mathbb{G}\left(\theta^{i}, \mathbf{v}^{i}\right)$ is an isomorphism of $\mathbb{X}$.

We start by proving the following continuity property using the discrete implicit function defined in the theorem of Brezzi, Rappaz, and Raviart [15].

Lemma 1 For any $\left(\theta_{N}, \mathbf{v}_{N}, \mathbf{w}_{N}\right)$ belonging to $\mathbb{T}_{N} \times \mathbb{V}_{N} \times \mathbb{V}_{N}$,

$$
\left|Z\left(\theta_{N}, \mathbf{v}_{N} ; \mathbf{w}_{N}\right)\right| \leq c \ln (N)^{\frac{1}{2}}\left\|\theta_{N}\right\|_{H(\mathbf{c u r l}, \Omega)}\left\|\mathbf{v}_{N}\right\|_{L^{2}(\Omega)^{2}}\left\|\mathbf{w}_{N}\right\|_{L^{2}(\Omega)^{2}},
$$

where $c$ is a positive constant.

Proof Using the Hölder inequality for all $r>2$ and $s>2$ such that $\frac{1}{r}+\frac{1}{s}=\frac{1}{2}$, we obtain

$$
\left|Z\left(\theta_{N}, \mathbf{v}_{N} ; \mathbf{w}_{N}\right)\right| \leq\left\|\theta_{N}\right\|_{L^{r}(\Omega)^{2}}\left\|\mathbf{v}_{N}\right\|_{L^{s}(\Omega)^{2}}\left\|\mathbf{w}_{N}\right\|_{L^{2}(\Omega)^{2}} .
$$

Then by the inverse inequality (see [29])

$$
\forall u_{N} \in \mathbb{P}_{N}(\Omega), \quad\left\|u_{N}\right\|_{L^{s}(\Omega)} \leq c N^{\left(2-\frac{4}{s}\right)}\left\|u_{N}\right\|_{L^{2}(\Omega)},
$$

and the fact that the embedding of $H(\operatorname{curl}, \Omega)$ into $L^{S}(\Omega)$ is continuous with a norm bounded by $s^{\frac{1}{2}}$ (see [30]), we get inequality (21) when $s=\ln (N)$.

Let $\mathfrak{L}$ the space of linear operators from $\mathbb{X}$ into $\mathbb{X}$.

Lemma 2 Under Assumption 2, there exists an integer $N^{*}$ such that, for all $N \geq N^{*}$, the operator Id $+\mathbb{S}_{N} D \mathbb{G}_{N}\left(\theta^{i}, \mathbf{v}^{i}\right)$ is an isomorphism of $\mathbb{X}$. The norm of its inverse operator is bounded independently of $N$. 
Proof Writing

$$
\begin{aligned}
\mathrm{Id}+\mathbb{S}_{N} D \mathbb{G}_{N}\left(\theta^{i}, \mathbf{v}^{i}\right)= & \operatorname{Id}+\mathbb{S} D \mathbb{G}\left(\theta^{i}, \mathbf{v}^{i}\right)-\left(\mathbb{S}-\mathbb{S}_{N}\right) D \mathbb{G}\left(\theta^{i}, \mathbf{v}^{i}\right) \\
& -\mathbb{S}_{N}\left(D \mathbb{G}\left(\theta^{i}, \mathbf{v}^{i}\right)-D \mathbb{G}_{N}\left(\theta^{i}, \mathbf{v}^{i}\right)\right),
\end{aligned}
$$

we prove the desired result in three steps.

1) The last term in the right-hand side of equality (22) is equal zero since $D \mathbb{G}\left(\theta^{i}, \mathbf{v}^{i}\right)=$ $D \mathbb{G}_{N}\left(\theta^{i}, \mathbf{v}^{i}\right)$ (see the definition of $\mathbb{G}$ and $\mathbb{G}_{N}$ ).

2) The second term in the right-hand side of equality (22) tends to zero as $N$ tends to infinity. For any $(\vartheta, \omega)$, we have

$$
D \mathbb{G}(\theta, \mathbf{v}) \cdot(\vartheta, \omega)=\theta \times \omega+\vartheta \times \mathbf{v} .
$$

Then from the property $[9,(2.37)]$ we obtain

$$
\operatorname{curl}(D \mathbb{G}(\theta, \mathbf{v}) \cdot(\vartheta, \omega))=\operatorname{grad}(\theta) \times \omega+\operatorname{grad}(\vartheta) \times \mathbf{v}
$$

We conclude that the term $\mathbb{S} D \mathbb{G}(\theta, \mathbf{v}) \cdot(\vartheta, \omega)$ is in $H^{2}(\Omega) \times H^{1}(\Omega)^{2}$ and satisfies

$$
\|\mathbb{S} D \mathbb{G}(\theta, \mathbf{v}) \cdot(\vartheta, \omega)\|_{H^{2}(\Omega) \times H^{1}(\Omega)^{2}} \leq c\left(\|\theta\|_{H^{2}(\Omega)}+\|\mathbf{v}\|_{H^{1}(\Omega)^{2}}\right)\|(\vartheta, \omega)\|_{\mathbb{X}} \cdot
$$

Finally, from (20) we have

$$
\left\|\left(\mathbb{S}-\mathbb{S}_{N}\right) D \mathbb{G}(\theta, \mathbf{v})\right\|_{\mathfrak{L}}=0 .
$$

(3) Using Assumption 2, take $\eta=\left\|(\operatorname{Id}+\mathbb{S} D \mathbb{G}(\theta, \mathbf{v}))^{-1}\right\|_{\mathfrak{L}}$ for $N$ large enough. Then the quantity in (23) is bounded by $\frac{1}{2 \eta}$, which gives the desired result with the norm of the inverse bounded by $2 \eta$.

Now we prove the following Lipschitz property of the operator $\mathbb{S}_{N}$.

Lemma 3 There exists a constant $k>0$ such that for any $(\hat{\theta}, \hat{\mathbf{v}}) \in \mathbb{X}$,

$$
\left\|\mathbb{S}_{N}\left(D \mathbb{G}_{N}(\theta, \mathbf{v})-D \mathbb{G}_{N}(\hat{\theta}, \hat{\mathbf{v}})\right)\right\|_{\mathfrak{L}} \leq k\left(\|\theta-\hat{\theta}\|_{H(\mathbf{c u r l}, \Omega)}+\|\mathbf{v}-\hat{\mathbf{v}}\|_{L^{2}(\Omega)^{2}}\right) .
$$

Proof For any $\mathbf{w}_{N} \in K_{N}$, we have

$$
\prec\left(D \mathbb{G}_{N}(\theta, \mathbf{v})-D \mathbb{G}_{N}(\hat{\theta}, \hat{\mathbf{v}})\right) \cdot(\vartheta, \omega), \mathbf{w}_{N} \succ=K\left(\theta-\hat{\theta}, \omega ; \mathbf{w}_{N}\right)+K\left(\vartheta, \mathbf{v}-\hat{\mathbf{v}} ; \mathbf{w}_{N}\right) .
$$

This leads to the desired Lipschitz property using the same idea as in the proof of Lemma 1 and (19).

Lemma 4 Assume that the data function $\mathbf{f}$ belongs to the space $L^{2}\left(0, T ; H^{\mu}(\Omega)^{2}\right), \mu>\frac{3}{2}$, and that the solution $\left(\theta^{i}, \mathbf{v}^{i}, p^{i}\right), 1 \leq i \leq I$, of problem (7)-(8) belongs to $H^{s+1}(\Omega) \times H^{s}(\Omega)^{2} \times$ $H^{s}(\Omega), s>1$. We have the following estimate:

$$
\begin{aligned}
\left\|\left(\theta^{i}, \mathbf{v}^{i}\right)+\mathbb{S}_{N} \mathbb{G}_{N}\left(\theta^{i}, \mathbf{v}^{i}\right)\right\|_{\mathbb{X}} \leq & c\left(\left(|h|+N^{1-s}\right)\left(\left\|\theta^{i}\right\|_{\left.H^{s+1} \Omega\right)}+\left\|\mathbf{v}^{i}\right\|_{H^{s}(\Omega)^{2}}\right)\right. \\
& \left.+\left(|h|+N^{-\mu}\right)\|\mathbf{f}\|_{L^{2}\left(0, T ; H^{\mu}(\Omega)^{2}\right)}\right)
\end{aligned}
$$

with a constant $c$ only depending on the data $\mathbf{f}$ and $\mathbf{v}^{j}, 0 \leq j \leq i-1$. 
Proof From equality (17) we derive that

$$
\begin{aligned}
\left\|\mathrm{Id}+\mathbb{S}_{N} \mathbb{G}_{N}\left(\theta^{i}, \mathbf{v}^{i}\right)\right\|_{\mathbb{X}} \leq & \left\|\left(\mathbb{S}-\mathbb{S}_{N}\right) \mathbb{G}_{N}\left(\theta^{i}, \mathbf{v}^{i}\right)\right\|_{\mathbb{X}} \\
& +\left\|\mathbb{S}_{N}\left(\mathbb{G}_{N}\left(\theta^{i}, \mathbf{v}^{i}\right)-\mathbb{G}\left(\theta^{i}, \mathbf{v}^{i}\right)\right)\right\|_{\mathbb{X}}
\end{aligned}
$$

Based on (20), we bound the first term in the right-hand side of inequality (25). To bound the second term, we write, thanks to the definition of $\mathbb{G}$ and $\mathbb{G}_{N}$,

$$
\begin{aligned}
\prec & \left(\mathbb{G}_{N}\left(\theta^{i}, \mathbf{v}^{i}\right)-\mathbb{G}\left(\theta^{i}, \mathbf{v}^{i}\right)\right), \mathbf{w}_{N} \succ \\
& =L_{N}\left(\mathbf{w}_{N}\right)-L\left(\mathbf{w}_{N}\right) \\
& =\left(\mathbf{v}_{N}^{i-1}, \mathbf{w}_{N}\right)_{N}-\left(\mathbf{v}^{i-1}, \mathbf{w}_{N}\right)+h_{i}\left(\left(\mathrm{I}_{N}\left(\mathbf{f}^{i}\right), \mathbf{w}_{N}\right)_{N}-\left(\mathbf{f}^{i}, \mathbf{w}_{N}\right)\right) .
\end{aligned}
$$

The approximation properties of the operators $\Pi_{N-1}$ and $\mathrm{I}_{N}$ (see [28]) give the bound for this last term, which concludes the proof of (24).

Consequently, in the following theorem, we state an error estimate.

Theorem 2 Assume that the data function $\mathbf{f}$ belongs to the space $L^{2}\left(0, T ; H^{\mu}(\Omega)^{2}\right), \mu>\frac{3}{2}$, and that the solution $\left(\theta^{i}, \mathbf{v}^{i}, p^{i}\right), 1 \leq i \leq I$, of problem (7)-(8) belongs to $H^{s+1}(\Omega) \times H^{s}(\Omega)^{2} \times$ $H^{s}(\Omega), s>1$, and satisfies Assumption 2. Then, there exist an integer $N^{*}$ and a positive real number $h^{*}$ such that for all $N \geq N^{*}$ and $|h| \leq h^{*}$, problem (15) has a unique solution. Moreover, this solution satisfies for all $1 \leq i \leq I$ the following error estimate:

$$
\begin{aligned}
& \left\|\theta^{i}-\theta_{N}^{i}\right\|_{H(\mathbf{c u r l}, \Omega)}+\left\|\mathbf{v}^{i}-\mathbf{v}_{N}^{i}\right\|_{H(\operatorname{div}, \Omega)}+\ln (N)^{-\frac{1}{2}}\left\|p^{i}-p_{N}^{i}\right\|_{L^{2}(\Omega)} \\
& \leq c\left(\left(|h|+N^{1-s}\right)\left(\left\|\theta^{i}\right\|_{\left.H^{s+1} \Omega\right)}+\left\|\mathbf{v}^{i}\right\|_{H^{s}(\Omega)^{2}}+\left\|p^{i}\right\|_{H^{s}(\Omega)}\right)\right. \\
& \left.\quad+\left(|h|+N^{-\mu}\right)\|\mathbf{f}\|_{L^{2}\left(0, T ; H^{\mu}(\Omega)^{2}\right)}\right) .
\end{aligned}
$$

Proof Using Lemmas 2-4 together with the Brezzi-Rappaz-Raviart theorem (see [15]), for $N$ large enough, we obtain that for all $1 \leq i \leq I$, problem (16) has a unique solution $\left(\theta_{N}^{i}, \mathbf{v}_{N}^{i}\right)$, which satisfies

$$
\begin{aligned}
\left\|\theta^{i}-\theta_{N}^{i}\right\|_{H(\mathbf{c u r}, \Omega)}+\left\|\mathbf{v}^{i}-\mathbf{v}_{N}^{i}\right\|_{H(\operatorname{div}, \Omega)} \leq & c\left(\left(|h|+N^{1-s}\right)\left(\left\|\theta^{i}\right\|_{\left.H^{s+1} \Omega\right)}+\left\|\mathbf{v}^{i}\right\|_{H^{s}(\Omega)^{2}}\right)\right. \\
& \left.+\left(|h|+N^{-\mu}\right)\|\mathbf{f}\|_{L^{2}\left(0, T ; H^{\mu}(\Omega)^{2}\right)}\right) .
\end{aligned}
$$

Besides, using the discrete inf-sup condition (14), for all $1 \leq i \leq I$, there exists a unique pressure $p_{N}^{i}$ in $\mathbb{M}_{N}$ such that

$$
\forall \mathbf{w}_{N} \in \mathbb{V}_{N}, \quad b_{N}\left(\mathbf{w}_{N}, p_{N}^{i}\right)=\left(\frac{1}{h_{i}}\right)\left(L_{N}\left(\mathbf{w}_{N}\right)-\hat{l}_{N}\left(\theta_{N}^{i}, \mathbf{v}_{N}^{i} ; \mathbf{w}_{N}\right)\right)-Z\left(\theta_{N}^{i}, \mathbf{v}_{N}^{i} ; \mathbf{w}_{N}\right) .
$$

Furthermore, for any $q_{N} \in \mathbb{M}_{N}$, having

$$
\begin{aligned}
b_{N}\left(\mathbf{w}_{N}, p_{N}^{i}-q_{N}\right)= & b\left(\mathbf{w}_{N}, p^{i}-q_{N}\right)-L\left(\mathbf{w}_{N}\right)+L_{N}\left(\mathbf{w}_{N}\right)+\hat{l}\left(\theta^{i}-\theta_{N}^{i}, \mathbf{v}^{i}-\mathbf{v}_{N}^{i} ; \mathbf{w}_{N}\right) \\
& +\left(\hat{l}-\hat{l}_{N}\right)\left(\theta_{N}^{i}, \mathbf{v}_{N}^{i} ; \mathbf{w}_{N}\right)+Z\left(\theta^{i}, \mathbf{v}^{i} ; \mathbf{w}_{N}\right)-Z\left(\theta_{N}^{i}, \mathbf{v}_{N}^{i} ; \mathbf{w}_{N}\right),
\end{aligned}
$$

we deduce the estimate for $\left\|p^{i}-p_{N}^{i}\right\|_{L^{2}(\Omega)}$ from (14) and the triangle inequality. 
Remark 2 Estimate (26) is fully optimal for the vorticity and velocity, whereas it is quasioptimal for the pressure.

\section{Resolution algorithm and numerical results}

\subsection{Resolution algorithm}

Considering the result of the error estimate, we will establish numerical tests just in the two-dimensional case on the square $\Omega=]-1,1{ }^{2}$. We elaborate the following iterative algorithm to solve problem (12). For simplicity, we omit the indices $N$.

Step 1. We start by solving the linear Stokes problem:

If $\mathbf{v}^{0}=\mathrm{I}_{N}\left(\mathbf{v}_{0}\right)$, then knowing $\mathbf{v}_{0}^{i-1}$, find $\left(\theta_{0}^{i}, \mathbf{v}_{0}^{i}, p_{0}^{i}\right)$ in $\mathbb{T}_{N} \times \mathbb{V}_{N} \times \mathbb{M}_{N}$ such that for $1 \leq i \leq I$,

$$
\begin{aligned}
& \forall \mathbf{w}_{N} \in \mathbb{V}_{N}, \quad \hat{l}_{N}\left(\theta_{0}^{i}, \mathbf{v}_{0}^{i} ; \mathbf{w}_{N}\right)+h_{i} b_{N}\left(\mathbf{w}_{N}, p_{0}^{i}\right)=L_{N}\left(\mathbf{w}_{N}\right), \\
& \forall q_{N} \in \mathbb{M}_{N}, \quad b_{N}\left(\mathbf{v}_{0}^{i}, q_{N}\right)=0, \\
& \forall \vartheta_{N} \in \mathbb{T}_{N}, \quad t_{N}\left(\theta_{0}^{i}, \mathbf{v}_{0}^{i} ; \boldsymbol{\vartheta}_{N}\right)=0 .
\end{aligned}
$$

Step 2. We suppose that the $(k-1)$ th iteration $\left(\theta_{k-1}^{i}, \mathbf{v}_{k-1}^{i}, p_{k-1}^{i}\right)$ is known. Then we solve the problem:

$$
\begin{aligned}
& \text { If } \mathbf{v}^{0}=\mathrm{I}_{N}\left(\mathbf{v}_{0}\right) \text {, then knowing } \mathbf{v}_{k}^{i-1}, \text { find }\left(\theta_{k}^{i}, \mathbf{v}_{k}^{i}, p_{k}^{i}\right) \text { in } \mathbb{T}_{N} \times \mathbb{V}_{N} \times \mathbb{M}_{N} \text { such that for } 1 \leq i \leq I, \\
& \begin{aligned}
& \forall \mathbf{w}_{N} \in \mathbb{V}_{N}, \\
& \hat{l}_{N}\left(\theta_{k}^{i}, \mathbf{v}_{k}^{i} ; \mathbf{w}_{N}\right)+h_{i} Z_{N}\left(\theta_{k-1}^{i}, \mathbf{v}_{k}^{i} ; \mathbf{w}_{N}\right)+h_{i} Z_{N}\left(\theta_{k}^{i}, \mathbf{v}_{k-1}^{i} ; \mathbf{w}_{N}\right)+h_{i} b_{N}\left(\mathbf{w}_{N}, p_{k}^{i}\right) \\
& \quad L_{N}\left(\mathbf{w}_{N}\right)+h_{i} Z_{N}\left(\theta_{k-1}^{i}, \mathbf{v}_{k-1}^{i} ; \mathbf{w}_{N}\right), \\
& \forall q_{N} \in \mathbb{M}_{N}, \quad b_{N}\left(\mathbf{v}_{k}^{i}, q_{N}\right)=0, \\
& \forall \vartheta_{N} \in \mathbb{T}_{N}, \quad t_{N}\left(\theta_{k}^{i}, \mathbf{v}_{k}^{i} ; \boldsymbol{\vartheta}_{N}\right)=0 .
\end{aligned}
\end{aligned}
$$

We do the iterations until the following condition is satisfied:

$$
\left(\left\|\theta_{k}^{i}-\theta_{k-1}^{i}\right\|_{H(\mathbf{c u r l}, \Omega)}^{2}+\left\|\mathbf{v}_{k}^{i}-\mathbf{v}_{k-1}^{i}\right\|_{H(\mathrm{div}, \Omega)}^{2}\right)^{\frac{1}{2}} \leq \xi
$$

for some fixed $\xi$.

In the following, we start by presenting the linear system deduced from the discrete problem (27). We build a basis of the discrete spaces $\mathbb{T}_{N}, \mathbb{V}_{N}$, and $\mathbb{M}_{N}$.

We consider the Lagrange polynomial $\psi_{p}$ in $\mathbb{P}_{N}(-1,1)$ associated with the nodes $\epsilon_{p}$, $0 \leq p \leq N$, such that

$$
\psi_{p} \in \mathbb{P}_{N}([-1,1]), \quad \psi_{p}\left(\epsilon_{q}\right)=\delta_{p q}, \quad 0 \leq p, q \leq N
$$

where $\delta_{p q}$ is the Kronecker symbol. We fix the integer $p^{*}$ between 0 and $N$ equal to $\frac{N}{2}$ or to $\frac{(N+1)}{2}$. We denote the set $P^{*}=\{0, \ldots, N\} \backslash\left\{p^{*}\right\}$ and consider the polynomial $\psi_{p}^{*} \in$ $\mathbb{P}_{N-1}([-1,1])$ such that

$$
\psi_{p}^{*}(\zeta)=\psi_{p}(\zeta) \frac{\epsilon_{p}-\epsilon_{p^{*}}}{\zeta-\epsilon_{p^{*}}}, \quad p \in P^{*}
$$


Then the discrete unknowns $\theta_{N}^{i}, \mathbf{v}_{N}^{i}$ and a pseudopressure $\breve{p}_{N}^{i}$ are written as

$$
\begin{aligned}
\theta_{N}^{i}(x, y) & =\sum_{p=1}^{N-1} \sum_{q=1}^{N-1} \theta_{p q}^{i} \psi_{p}(x) \psi_{q}(y), \\
\mathbf{v}_{N x}^{i}(x, y) & =\sum_{p=1}^{N-1} \sum_{q \in P^{*}} \mathbf{v}_{p q}^{i x} \psi_{p}(x) \psi_{q}^{*}(y), \quad \mathbf{v}_{N y}^{i}(x, y)=\sum_{p \in P^{*}} \sum_{q=1}^{N-1} \mathbf{v}_{p q}^{i j} \psi_{p}^{*}(x) \psi_{q}(y), \\
\breve{p}_{N}^{i}(x, y) & =\sum_{p \in P^{*}, q \in P^{*},(p, q) \neq(0,0)} \breve{p}_{p q} \psi_{p}^{*}(x) \psi_{q}^{*}(y) .
\end{aligned}
$$

The function $\breve{p}^{i}$ does not belong to $L_{0}^{2}(\Omega)$. However, the real pressure $p_{N}^{i}$ is obtained from the formula

$$
p_{N}^{i}(x, y)=\breve{p}_{N}^{i}(x, y)-\frac{1}{4}\left(\breve{p}_{N}^{i}, 1\right)_{N} .
$$

The components of the unknowns $\theta_{N}^{i}, \mathbf{v}_{N x}^{i}, \mathbf{v}_{N y}^{i}$, and $p_{N}^{i}$ allow us to form the unknowns vectors denoted by $\Sigma^{i}, V_{x}^{i}, V_{y}^{i}$, and $P^{i}$. Their dimensions are equal to $(N-1)^{2}, N(N-1)$, $N(N-1)$, and $N^{2}-1$, respectively. We consider $V^{0}=\left(V_{1}^{0}, V_{2}^{0}\right)$, where the components of the vectors $V_{1}^{0}$ and $V_{2}^{0}$ are respectively $v_{N x}^{0}\left(\epsilon_{p}, \epsilon_{q}\right)$ and $v_{N y}^{0}\left(\epsilon_{p}, \epsilon_{q}\right)$ such that $\mathbf{v}^{0}=\left(v_{N x}^{0}, v_{N y}^{0}\right)$. Consequently, we formulate the discrete problem (27) as the following equivalent linear system:

$$
\left(\begin{array}{cccc}
v A_{1}^{i}-D_{11}^{i} & 0 & -D_{21}^{i} & -B_{1}^{i} \\
v A_{2}^{i}-D_{12}^{i} & D_{22}^{i} & 0 & -B_{2}^{i} \\
0 & B_{1}^{i T} & B_{2}^{i T} & 0 \\
C_{\omega} & -A_{1}^{i T} & -A_{2}^{i T} & 0
\end{array}\right)\left(\begin{array}{c}
\Sigma^{i} \\
V_{x}^{i} \\
V_{y}^{i} \\
P^{i}
\end{array}\right)=\left(\begin{array}{c}
F_{1}^{i}+N_{1}^{i} \\
F_{2}^{i}+N_{2}^{i} \\
0 \\
0
\end{array}\right)
$$

where $B^{T}$ is the transpose of a matrix $B$. The matrices $A^{i}=\left(A_{1}^{i}, A_{2}^{i}\right), B^{i}=\left(B_{1}^{i}, B_{2}^{i}\right)$, and $C_{\omega}$ are the same as for the Stokes problem (see [10, Sect. 6]).

The matrices $D_{1}=\left(D_{11}, D_{12}\right), D_{2}=\left(D_{21}, D_{22}\right)$, and $N_{1}=\left(N_{1}, N_{2}\right)$ are made respectively from the terms $Z_{N}\left(\theta_{1}^{i}, \mathbf{v}_{0}^{i} ; \mathbf{v}_{N}\right)$., $Z_{N}\left(\theta_{0}^{i}, \mathbf{v}_{1}^{i} ; \mathbf{v}_{N}\right)$., and $Z_{N}\left(\theta_{0}^{i}, \mathbf{v}_{0}^{i} ; \mathbf{v}_{N}\right)$.

Since the global matrix of the linear system (28) is not symmetric, the GMRES method [16] is used for the resolution.

\subsection{Numerical results}

In this section, we start by studying the time convergence. We consider a given solution obtained from the formulas $\mathbf{v}=\operatorname{curl} \varphi$ and $\theta=\operatorname{curl} \mathbf{v}$, where $\varphi$ is the stream function. We handle the following two cases.

Case (1): Assume that the steam function $\varphi$ and the pressure $p$ are $\mathcal{C}^{\infty}$ are related to the time and space so that $(\theta, \mathbf{v} ; p)$ is a solution of problem (4):

$$
\varphi(t, x, y)=e^{t} \sin (\pi x) \sin (\pi y), \quad p(t, x, y)=e^{-t}(x+y) .
$$

Case (2): Assume that the steam function $\varphi$ and the pressure $p$ are less regularly related to the time and space so that $(\theta, \mathbf{v} ; p)$ is a solution of problem (4):

$$
\varphi(t, x, y)=t^{\frac{1}{2}}\left(1-x^{2}\right)^{3}\left(1-y^{2}\right)^{\frac{7}{2}}, \quad p(t, x, y)=t^{\frac{1}{2}} x\left(1-x^{2}\right)^{\frac{3}{2}}\left(1+y^{2}\right)^{-\frac{1}{2}} .
$$




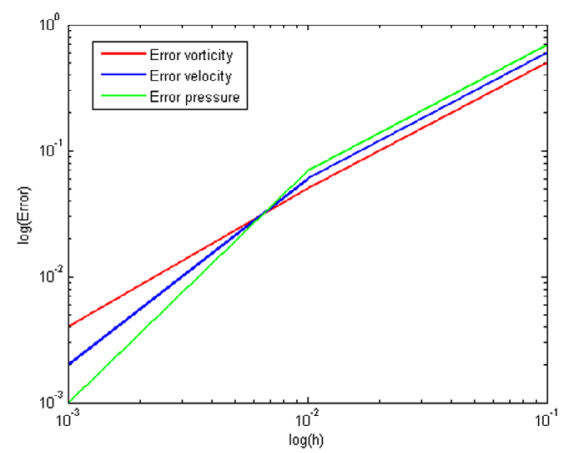

(a) Regular solution defined in (29)

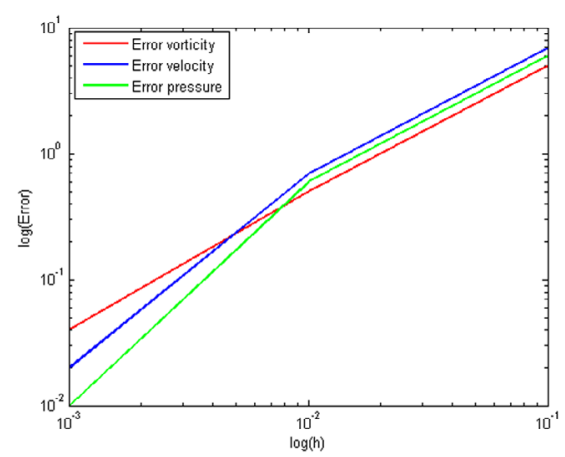

(b) Singular solution defined in (30)

Figure 1 The time convergence

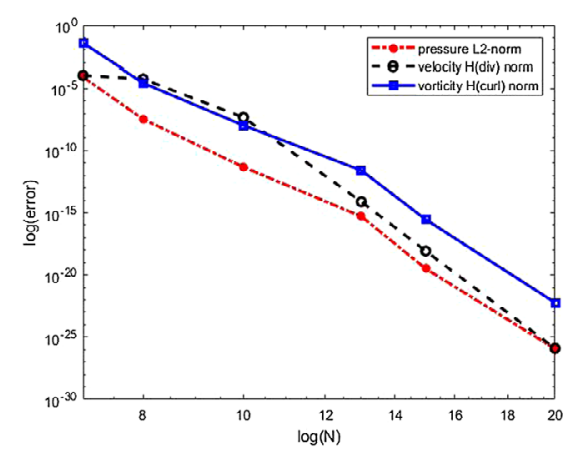

(a) Solution defined in (28)

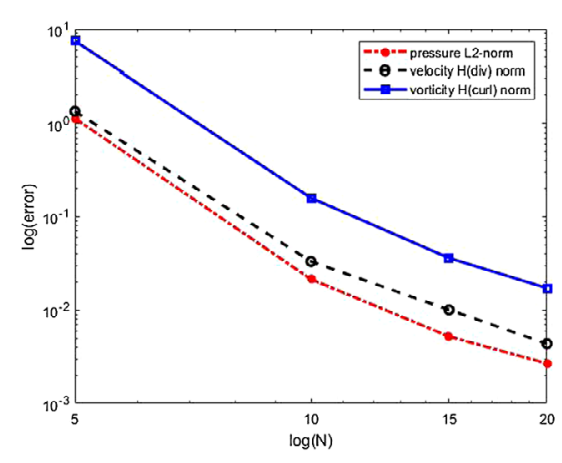

(b) Solution defined in (29)

Figure 2 The spectral convergence

The velocity is a Gaussian; then $\mathbf{v}_{0}=\mathbf{v}(\mathbf{x}, 0)=(0,0)$. We fix the space discrete parameter $N=40, T=1$, and the time steps $h$ varying in $\{0.1,0.001,0.0001\}$. The viscosity $v$ and e tolerance $\xi$ are given by

$$
\nu=5 \cdot 10^{-2}, \quad \xi=10^{-12} .
$$

Figure 1 presents the curves of convergence for the three terms $\log \left\|\theta-\theta_{N}^{i}\right\|_{H(\mathrm{curl}, \Omega)}$ (in red), $\log \left\|\mathbf{v}-\mathbf{v}_{N}^{i}\right\|_{H(\mathrm{div}, \Omega)}$ (in blue), and $\log \left\|p-p_{N}^{i}\right\|_{L^{2}(\Omega)}$ (in green) as functions of $\log (h)$. Figures 1 (a) and 1(b) represent the convergence in time for the continuous solutions defined in (29) and (30), respectively. We remark that in the two cases (regular solution or less regular solution) the time convergence order is almost equal to 1 , which confirms the result of Theorem 2.

In Fig. 2(a), for the solution issued from (29), we present the spectral convergence curves on the vorticity $\theta$ in norm $H(\operatorname{curl}, \Omega)$, the velocity $\mathbf{v}$ in norm $H(\operatorname{div}, \Omega)$, and the pressure in norm $L^{2}(\Omega)$. These error curves are provided in semilogarithmic scales, as functions of $\log (N)$, for $N$ varying from 5 to 30 . As can be expected from Theorem 2 , the convergence is exponential for the solution, and the slope for the error curve on the pressure is the same as that for the vorticity and velocity. 


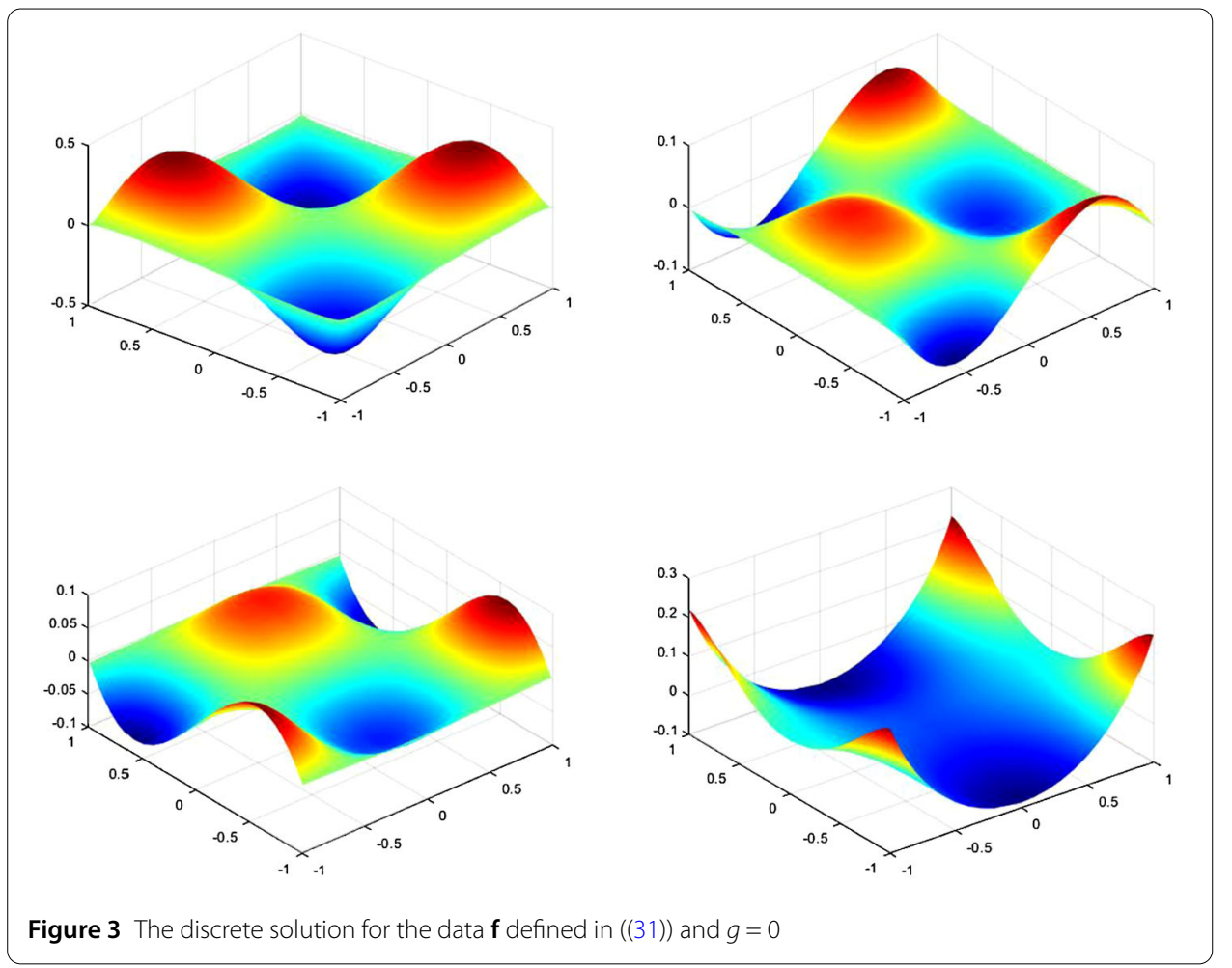

Figure 2(b) shows the convergence curves of the relative errors of the vorticity $\theta$ in norm $H(\mathbf{c u r l}, \Omega)$, the velocity $\mathbf{v}$ in norm $H(\operatorname{div}, \Omega)$, and the pressure in norm $L^{2}(\Omega)$ in semilogarithmic scales, as functions of $\log (N)$, for the solution issued from (30). We note that the error is much larger for the singular solution (30) than that for the regular solution (29), which confirms the results of Theorem 2.

Figure 3 corresponds from top to bottom and left to right to the discrete vorticity, the two components of the discrete velocity, and the discrete pressure for the data

$$
\mathbf{f}=\left(f_{x}, f_{y}\right)=\left(t x y^{2}, 0\right), \quad \mathbf{v}_{0}=(0,0)
$$

homogeneous boundary conditions $\mathbf{v} \cdot \mathbf{n}=g=0$ on $\Gamma$, and $N=35$.

Now we handle the influence of the viscosity $v$ on the number of iterations. We take $\xi=10^{-12}$ and the regular solution issued from (29).

Figure 4 presents the number of iterations processed by the algorithm as a function of $N$ as it varies from 5 to 25 . This number of iterations is greater when the viscosity $v$ decreases.

We further use a nonhomogeneous test. We deal with a Poiseuille linear flow, the data $\mathbf{f}=(0,0)$, and the boundary condition $\mathbf{v} \cdot \mathbf{n}=g$ given by

$$
\begin{aligned}
& g(-1, y)=-y(1-y), \quad \text { if } 0<y<1, \\
& g(1, y)=y(1+y), \quad \text { if }-1<y<0, \\
& \text { else, } g(x, y)=0 .
\end{aligned}
$$

Figure 5 presents from left to right the curves of the velocity field with viscosity $v=10^{-1}$ obtained with $N=20$ and with viscosity $v=10^{-2}$ obtained with $N=30$. The vorticity and 


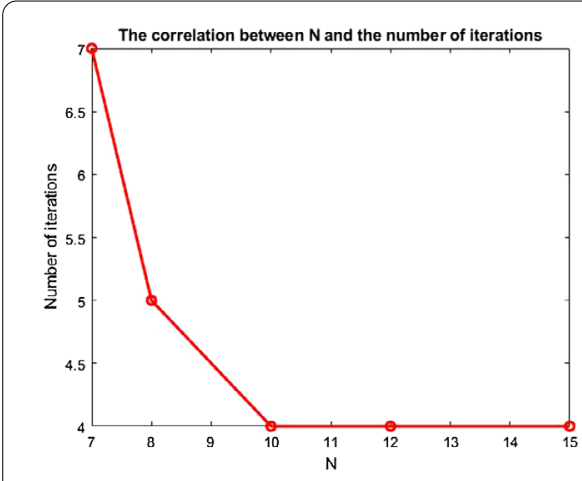

(a) Iterations for $v=10^{-1}$

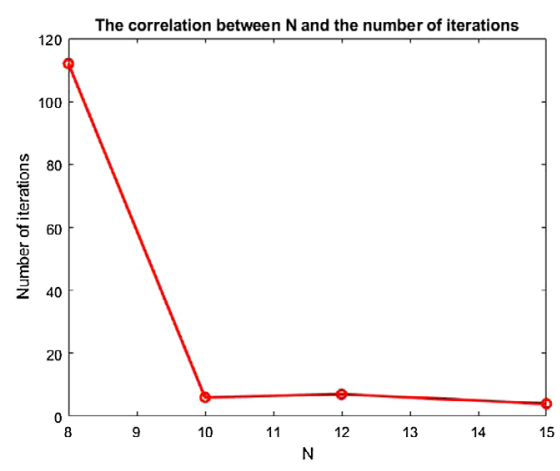

(b) Iterations for $v=10^{-2}$

Figure 4 Influence of the viscosity on the time convergence of the algorithm
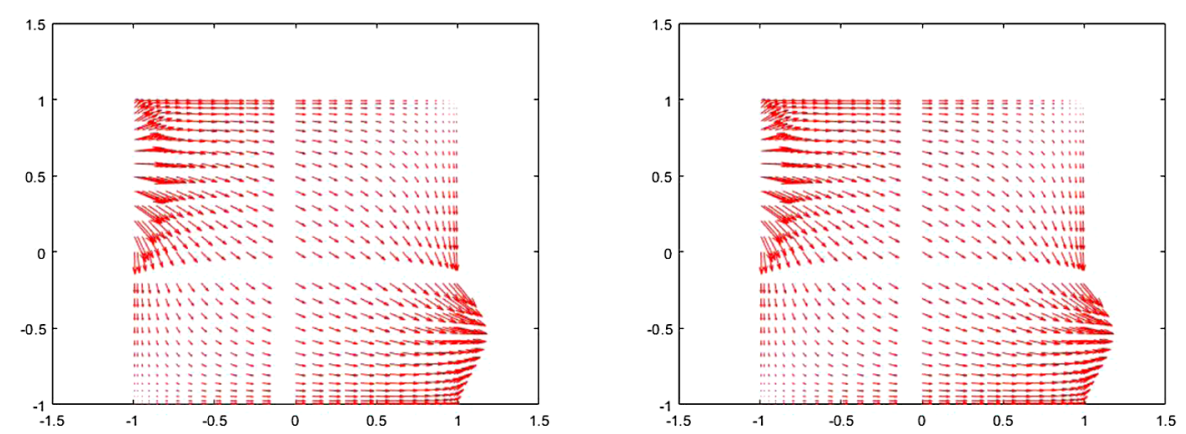

Figure 5 Curves of the velocity fields for different viscosities

pressure are equal to zero since the data $\mathbf{f}=(0,0)$, which is easily proved from the NavierStokes equations.

\section{Conclusion}

This paper deals with the resolution and implementation of the implicit Euler scheme in time and spectral discretization in space of the nonstationary vorticity velocity pressure formulation of the Navier-Stokes problem with nonstandard boundary conditions. We prove using the Brezzi-Rappaz-Raviart theorem that the new discrete formulation has a unique local solution. In dimension two, we show an optimal error estimate for the vorticity and velocity and a nearly optimal for the pressure,.

\section{Acknowledgements}

The authors would like to extend their sincere appreciation to the Deanship of Scientific Research at King Saud University for funding this Research group No. (RG-1435-026).

\section{Funding}

Not applicable

Availability of data and materials

Not applicable. 
Authors' contributions

The authors declare that the study was realized in collaboration with equal responsibility. All authors read and approved the final manuscript.

\section{Author details}

'Department of Mathematics, College of Sciences, King Saud University, Riyadh, Saudi Arabia. ${ }^{2}$ Information Technology

Department, College of Computer and Information Sciences, King Saud University, Riyadh, Saudi Arabia.

\section{Publisher's Note}

Springer Nature remains neutral with regard to jurisdictional claims in published maps and institutional affiliations.

\section{Received: 23 September 2020 Accepted: 15 October 2020 Published online: 23 October 2020}

\section{References}

1. Bègue, C., Conca, C., Murat, F., Pironneau, O.: Les équations de Stokes et de Navier-Stokes avec des conditions aux limites sur la pression. In: Brezis, H., Lions, J.-L. (eds.) Nonlinear Partial Differential Equations and Their Applications. Collège de France Seminar, vol. IX, pp. 179-264. Longman, Harlow (1988)

2. Dubois, F.: Vorticity-velocity-pressure formulation for the Stokes problem. Math. Methods Appl. Sci. 25, 1091-1119 (2002)

3. Salmon, S.: Développement numérique de la formulation tourbillon-vitesse-pression pour le problème de Stokes. PhD thesis, Université Pierre et Marie Curie, Paris, France (1999)

4. Amara, M., Capatina-Papaghiuc, D., Chacon-Vera, E., Trujillo, D.: Vorticity-velocity-pressure formulation for Navier-Stokes equations. Comput. Vis. Sci. 6, 47-52 (2004)

5. Dubois, F., Salaün, M., Salmon, S.: Vorticity-velocity-pressure and stream function-vorticity formulations for the Stokes problem. J. Math. Pures Appl. 82, 1395-1451 (2003)

6. Amara, M.D., Chacon-Vera, E., Trujillo, D.: Stabilized finite element method for the Navier-Stokes equations, with non standard boundary conditions. J. Sci. Comput. 18, 4-9 (2005)

7. Bernardi, C., Sayah, T.: A posteriori error analysis of the time dependent Stokes equations with mixed boundary conditions. IMA J. Numer. Anal. 35, 179-198 (2015)

8. Bernardi, C., Sayah, T.: A posteriori error analysis of the time dependent Navier-Stokes equations with mixed boundary conditions. SeMA J. 69, 1-23 (1991)

9. Azaïez, M., Bernardi, C., Chorfi, N.: Spectral discretization of the vorticity, velocity and pressure formulation of the Navier-Stokes equations. Numer. Math. 104, 1-26 (2006)

10. Bernardi, C., Chorfi, N.: Spectral discretization of the vorticity, velocity and pressure formulation of the Stokes problem. SIAM J. Numer. Anal. 44, 826-850 (2006)

11. Abdelwahed, M., Chorfi, N.: Spectral discretization of the time dependent vorticity velocity pressure formulation of the Stokes problem. Math. Methods Appl. Sci. 1-18 (2020)

12. Abdelwahed, M., Chorfi, N.: Spectral discretization of the time dependent vorticity velocity pressure formulation of the Navier-Stokes equations. Bound. Value Probl. 2020, 152 (2020)

13. Maday, Y., Ronquist, E.M.: Optimal error analysis of spectral methods with emphasis on non-constant coefficients and deformed geometries. Comput. Methods Appl. Mech. Eng. 80,91-115 (1990)

14. Amoura, K., Azaïez, M., Bernardi, C., Chorfi, N., Saad, S.: Spectral element discretization of the vorticity, velocity and pressure formulation of the Navier-Stokes problem. Calcolo 44, 165-188 (2007)

15. Brezzi, F., Rappaz, J., Raviart, P.-A.: Finite dimensional approximation of nonlinear problems. Part I: Branches of nonsingular solutions. Numer. Math. 36, 1-25 (1980)

16. Walker, H.F.: Implementation of the GNRES method using householder transformation. SIAM J. Sci. Stat. Comput. 9, 152-163 (1988)

17. Bernardi, C., Girault, V.: Espaces duaux des domaines des opérateurs divergence et rotationnel avec trace nulle. Internal Report, Laboratoire Jacques-Louis Lions, Université Pierre et Marie Curie, Paris, France (2003)

18. Amrouche, C., Bernardi, C., Dauge, M., Girault, V.: Vector potentials in three-dimensional nonsmooth domains. Math. Methods Appl. Sci. 21, 823-864 (1998)

19. Bernardi, C., Hecht, F., Verfurth, R.: Finite element discretization of the three-dimensional Navier-Stokes equations with mixed boundary conditions. Math. Model. Numer. Anal. 3, 1185-1201 (2009)

20. Girault, V., Raviart, P.-A.: Finite Element Methods for Navier-Stokes Equations, Theory and Algorithms. Springer, Berlin (1986)

21. Temam, R.: Navie-Stokes Equations. Theory and Numerical Analysis. Studies in Mathematics and Its Applications, vol. 2. North-Holland, Amsterdam (1977)

22. Girault, V., Raviart, P.-A.: Finite Element Approximation of the Navier-Stokes Equations. Lecture Notes in Mathematics, vol. 749. Springer, Berlin (1979)

23. Costabel, M., Dauge, M.: Espaces fonctionnels Maxwell: Les gentils, les méchants et les singularités (1998) http://perso.univ-rennes $1 . \mathrm{fr} / \mathrm{monique}$.dauge

24. Costabel, M., Dauge, M.: Computation of resonance frequencies for Maxwell equations in non-smooth domains. In: Ainsworth, M., Davies, P., Duncan, D., Martin, P., Rynne, B. (eds.) Topics in Computational Wave Propagation. Springer, Berlin (2004)

25. Wang, Y., Xiao, J.: Well/ill-posedness for the dissipative Navier-Stokes system in generalized Carleson measure spaces. Adv. Nonlinear Anal. 1, 203-224 (2019)

26. Al Baba, H.: Maximal $P_{-}^{P} l^{9}$ regularity to the Stokes problem with Navier boundary conditions. Adv. Nonlinear Anal. 1 $743-761(2019)$

27. Nédélec, J.-C.: Mixed finite elements in $\mathbb{R}^{3}$. Numer. Math. 35, 315-341 (1980)

28. Bernardi, C., Maday, Y.: Spectral Method. In: Ciarlet, P.G., Lions, J.-L. (eds.) Handbook of Numerical Analysis. North-Holland, Amsterdam (1997) 
29. Bernardi, C., Dauge, M., Maday, Y.: Polynomials in the Sobolev world. Internal Report, Laboratoire Jacques-Louis Lions (2003)

30. Talenti, G.: Best constant in Sobolev inequality. Ann. Mat. Pura Appl. 110, 353-372 (1976)

Submit your manuscript to a SpringerOpen ${ }^{\circ}$ journal and benefit from:

- Convenient online submission

- Rigorous peer review

- Open access: articles freely available online

- High visibility within the field

- Retaining the copyright to your article

Submit your next manuscript at $\gg$ springeropen.com 